\title{
Modeling the spread of COVID-19 pandemic in Morocco*
}

Houssine Zine, El Mehdi Lotfi, Marouane Mahrouf, Adnane Boukhouima, Yassine Aqachmar, Khalid Hattaf, Delfim F. M. Torres and Noura Yousfi

\footnotetext{
Houssine Zine

Center for Research and Development in Mathematics and Applications (CIDMA), Department of Mathematics, University of Aveiro, 3810-193 Aveiro, Portugal, e-mail: zinehoussine@ua.pt

El Mehdi Lotfi

Laboratory of Analysis, Modeling and Simulation (LAMS), Faculty of Sciences Ben M'sik, Hassan II University of Casablanca, P.B 7955 Sidi Othman, Casablanca, Morocco, e-mail:lotfiimehdi@gmail.com

Marouane Mahrouf

Laboratory of Analysis, Modeling and Simulation (LAMS), Faculty of Sciences Ben M'sik, Hassan II University of Casablanca, P.B 7955 Sidi Othman, Casablanca, Morocco, e-mail: marouane.mahrouf@gmail.com

Adnane Boukhouima

Laboratory of Analysis, Modeling and Simulation (LAMS), Faculty of Sciences Ben M'sik, Hassan II University of Casablanca, P.B 7955 Sidi Othman, Casablanca, Morocco,

e-mail:adnaneboukhouima@gmail.com
}

Yassine Aqachmar

World Health Organization (WHO), Country Office, Rabat, Morocco, e-mail: aqachmary@who.int

Khalid Hattaf

Centre Régional des Métiers de l'Education et de la Formation (CRMEF), P.B 20340 Derb Ghalef, Casablanca, Morocco, e-mail: k.hattaf@yahoo.fr

Delfim F. M. Torres

Center for Research and Development in Mathematics and Applications (CIDMA),

Department of Mathematics, University of Aveiro, 3810-193 Aveiro, Portugal, e-mail: delfim@ ua.pt

Noura Yousfi

Laboratory of Analysis, Modeling and Simulation (LAMS), Faculty of Sciences Ben M'sik,

Hassan II University of Casablanca, P.B 7955 Sidi Othman, Casablanca, Morocco,

e-mail: nourayousfi.fsb@gmail.com 
Abstract Nowadays, coronavirus disease 2019 (COVID-19) poses a great threat to public health and economy worldwide. Unfortunately, there is yet no effective drug for this disease. For this, several countries have adopted multiple preventive interventions to avoid the spread of COVID-19. Here, we propose a delayed mathematical model to predict the epidemiological trend of COVID-19 in Morocco. Parameter estimation and sensitivity analysis of the proposed model are rigorously studied. Moreover, numerical simulations are presented in order to test the effectiveness of the preventive measures and strategies that were imposed by the Moroccan authorities and also help policy makers and public health administration to develop such strategies.

Keywords: COVID-19, coronavirus, mathematical modeling, basic reproduction number, prediction.

\section{Introduction}

Coronavirus disease 2019 (COVID-19) is an infectious disease that appeared in China at the end of 2019. It is caused by a new type of virus belonging to the coronaviruses family and recently named severe acute respiratory syndrome coronavirus 2 (SARSCoV-2) [1]. On March 11, 2020, COVID-19 was reclassified as a pandemic by the World Health Organization (WHO). The disease spreads rapidly from country to country, causing enormous economic damage and many deaths worldwide. The first case of COVID-19 in Morocco was confirmed on March 2, 2020 in city of Casablanca. It involved a Moroccan expatriate residing in Italy and who came from Italy on February 27, 2020. As of April 17, 2020, the confirmed cases reached 2564 and the number of recoveries reached 281 with a total number of 135 deaths [2].

Moroccan authorities have implemented multiple preventive measures and strategies to control the spread of disease, such as the closing of borders, suspension of schools and universities, closing coffee shops, the shut-down of all mosques in the country, etc. Further, Morocco has declared a state of health emergency during the period from March 20 to April 20, 2020, to avoid the spread of COVID-19. During this period, movement during the day should be limited to work, shopping, medical care, purchasing medicine, medical supplies, and emergency situations only. In addition, and from April 6, 2020, the wearing of a mask became compulsory for all persons authorized to move.

Mathematical modeling of COVID-19 transmission has attracted the attention of many scientists. Tang et al. [3] used a Susceptible-Exposed-Infectious-Recovered (SEIR) compartmental model to estimate the basic reproduction number of COVID19 transmission based on data obtained for the confirmed cases of the disease in mainland China. Wu et al. [4] provided an estimate of the size of the epidemic in Wuhan on the basis of the number of cases exported from Wuhan to cities

\footnotetext{
* This is a preprint of a paper whose final and definite form is published in Analysis of Infectious Disease Problems (Covid-19) and Their Global Impact, Springer Nature Singapore Pte Ltd. First submitted 18/April/2020; revised 22/Sept/2020; accepted 08/Oct/2020.
} 
outside mainland China by using a SEIR model. In [5], Kuniya applied the SEIR compartmental model for the prediction of the epidemic peak for COVID-19 in Japan by using the real-time data from January 15 to February 29, 2020. Fanelli and Piazza [6] analyzed and forecasted COVID-19 spreading in China, Italy and France by using a simple Susceptible-Infected-Recovered-Death (SIRD) model. The authors of [7] present a mathematical model and study the dynamics of COVID-19 that emerged recently in Wuhan, China. For a fractional (non-integer order) model see [8].

In the models cited above, the transmission of the disease was assumed to be instantaneous and therefore they are formulated by ordinary differential equations (ODEs), without time delays. In this study, we propose a mathematical model governed by delay differential equations (DDEs) to predict the epidemiological trend of COVID-19 in Morocco and taking into account multiple preventive measures and strategies implemented by Moroccan authorities, related to the confinement period between March 2 and June 20, 2020, in order to control the spread of disease. To do this, Section 2 deals with the formulation of the model. Section 3 is devoted to parameters estimation and sensitivity analysis. Forecast of COVID-19 spreading in Morocco is presented in Section 4. We end with a discussion of the results, in Section 5

\section{Formulation of the model}

Around the world, all the countries that are attacked by the COVID-19 have imposed several strategies, with different degrees, to fight against it, namely the reduction of some rights by adopting the quarantine method in order to prevent contacts between vulnerable and infected individuals, closing the geographical borders of the countries, and enforcing the capacity of the sanitary system. Similarly, the Kingdom of Morocco quickly followed all of the previous strategies when the pandemic was in its early stages.

Remark 1 The terms "susceptibility" and "vulnerability" are often used interchangeably for populations with disproportionate health burdens [9]. The distinction between vulnerability and susceptibility marks the difference between being intact but fragile-vulnerable and being injured and predisposed to compound additional harm-susceptible [10]. Here, we refer to "the potential to contract the COVID-19" as vulnerability, to emphasize the environmental nature of the disease.

After the first reported positive case in Morocco, March 2, 2020, the closing of schools and universities is done at March 16, 2020; the state of health emergency (containment) is imposed to contain the outbreak from March 20, 2020; and the closure of the borders is performed at March 24, 2020. Additionally, the face mask is obligatory used in the general population at April 6, 2020. Based on these preventive measures and strategies, we model the dynamics of the transmission of COVID-19 in Morocco by extending the classical SIR model. Precisely, the population is divided into eight classes, denoted by $V, I_{s}, I_{a}, F_{b}, F_{g}, F_{c}, R$ and $D$, where $V$ represents the 
vulnerable sub-population, which is not infected and has not been infected before, but is susceptible to develop the disease if exposed to the virus; $I_{s}$ is the symptomatic infected sub-population, which has not yet been treated, it transmits the disease, and outside of proper support it can progress to spontaneous recovery or death; $I_{a}$ is the asymptomatic infected sub-population who is infected but does not transmit the disease, it is not known by the health system and progresses spontaneously to recovery; $F_{b}, F_{g}$ and $F_{c}$ are the patients diagnosed, supported by the Moroccan health system and under quarantine, and subdivided into three categories: benign, severe, and critical forms, respectively. Finally, $R$ and $D$ are the recovered and died classes. The schematic diagram of our extended model is illustrated in Figure 1

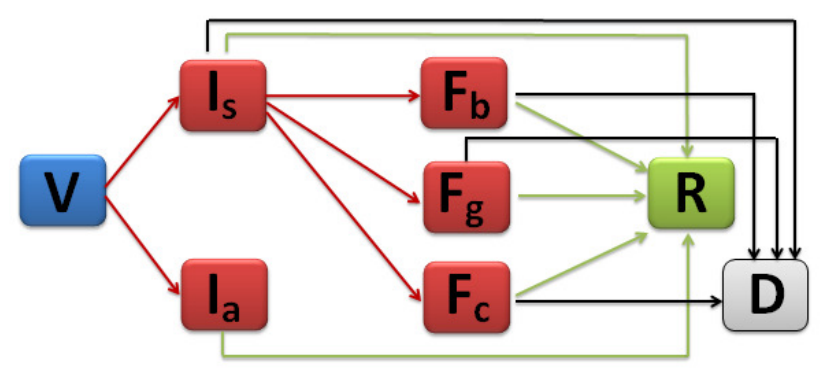

Fig. 1: Schematic diagram of our extended model.

Therefore, the extended model can be governed by the following system of DDEs:

$$
\left\{\begin{array}{l}
\frac{d V(t)}{d t}=-\beta(1-u) V(t) I_{s}(t), \\
\frac{d I_{s}(t)}{d t}=\beta \epsilon(1-u) V\left(t-\tau_{1}\right) I_{s}\left(t-\tau_{1}\right)-\left(\mu_{s}+\eta_{s}+\alpha\left(\gamma_{b}+\gamma_{g}+\gamma_{c}\right)\right) I_{s}(t), \\
\frac{d I_{a}(t)}{d t}=\beta(1-\epsilon)(1-u) V\left(t-\tau_{1}\right) I_{s}\left(t-\tau_{1}\right)-\eta_{a} I_{a}(t), \\
\frac{d F_{b}(t)}{d t}=\alpha \gamma_{b} I_{s}\left(t-\tau_{2}\right)-\left(\mu_{b}+r_{b}\right) F_{b}(t), \\
\frac{d F_{g}(t)}{d t}=\alpha \gamma_{g} I_{s}\left(t-\tau_{2}\right)-\left(\mu_{g}+r_{g}\right) F_{g}(t), \\
\frac{d F_{c}(t)}{d t}=\alpha \gamma_{c} I_{s}\left(t-\tau_{2}\right)-\left(\mu_{c}+r_{c}\right) F_{c}(t), \\
\frac{d R(t)}{d t}=\eta_{a} I_{a}(t)+\eta_{s} I_{s}(t)+r_{b} F_{b}(t)+r_{g} F_{g}(t)+r_{c} F_{c}(t), \\
\frac{d D(t)}{d t}=\mu_{s} I_{s}(t)+\mu_{b} F_{b}(t)+\mu_{g} F_{g}(t)+\mu_{c} F_{c}(t),
\end{array}\right.
$$


where $u$ represents the level of control strategies on the vulnerable population. We adopt the bilinear incidence rate to describe the infection of the disease and use parameter $\beta$ to denote the transmission rate. It is reasonable to assume that the infected individuals are subdivided into individuals with symptoms and others without symptoms, for which we employ the parameter $\epsilon$ to denote the proportion for the symptomatic individuals and $1-\epsilon$ for the asymptomatic ones. The parameter $\alpha$ measures the efficiency of public health administration for hospitalization. Diagnosed symptomatic infected population moves to the three forms: benign, severe and critical, by the rates $\gamma_{b}, \gamma_{g}$ and $\gamma_{c}$, respectively. The mean recovery period of these forms are denoted by $1 / r_{b}, 1 / r_{g}$ and $1 / r_{c}$, respectively. The later forms die also with the rates $\mu_{b}, \mu_{g}$ and $\mu_{c}$, respectively. Symptomatic infected population, which is not diagnosed, moves to the recovery compartment with a rate $\eta_{s}$ or dies with a rate $\mu_{s}$. On the other hand, asymptomatic infected population moves to the recovery compartment with a rate $\eta_{a}$. The times delay $\tau_{1}$ and $\tau_{2}$ denote the incubation period and the period time needed before hospitalization, respectively.

For biological reasons, we assume that the initial conditions of system (1) satisfy:

$$
\begin{aligned}
& V(\theta)=\phi_{1}(\theta) \geq 0, \quad I_{s}(\theta)=\phi_{2}(\theta) \geq 0, \quad I_{a}(\theta)=\phi_{3}(\theta) \geq 0, \\
& F_{b}(\theta)=\phi_{4}(\theta) \geq 0, \quad F_{g}(\theta)=\phi_{5}(\theta) \geq 0, \quad F_{c}(\theta)=\phi_{6}(\theta) \geq 0, \\
& R(\theta)=\phi_{7}(\theta) \geq 0, \quad D(\theta)=\phi_{8}(\theta) \geq 0, \quad \theta \in[-\tau, 0],
\end{aligned}
$$

where $\tau=\max \left\{\tau_{1}, \tau_{2}\right\}$. Let $C=C\left([-\tau, 0], \mathbb{R}^{8}\right)$ be the Banach space of continuous functions from the interval $[-\tau, 0]$ into $\mathbb{R}^{8}$, equipped with the uniform topology. It follows from the theory of functional differential equations [11] that system (1) with initial conditions $\left(\phi_{1}, \phi_{2}, \phi_{3}, \phi_{4}, \phi_{5}, \phi_{6}, \phi_{7}, \phi_{8}\right) \in C$ has a unique solution.

On the other hand, the basic reproduction number is an important threshold parameter that determines the spread of infection when the disease is introduced into the population [12]. This number is defined as the expected number of secondary cases produced, in a completely susceptible population, by a typical infective individual. By using the next generation matrix approach [13], the basic reproduction number $\mathcal{R}_{0}$ of system (1) is given by

$$
\mathcal{R}_{0}=\rho\left(F V^{-1}\right)=\frac{\beta \epsilon(1-u)}{\eta_{s}+\mu_{s}+\alpha\left(\gamma_{b}+\gamma_{g}+\gamma_{c}\right)},
$$

where $\rho$ is the spectral radius of the next generation matrix $F V^{-1}$ with

$$
F=\left(\begin{array}{cc}
\beta \epsilon(1-u) & 0 \\
0 & 0
\end{array}\right) \quad \text { and } \quad V=\left(\begin{array}{cc}
\eta_{s}+\mu_{s}+\alpha\left(\gamma_{b}+\gamma_{g}+\gamma_{c}\right) & 0 \\
0 & \eta_{a}
\end{array}\right) .
$$

\section{Parameter estimation and sensitivity analysis}

Based on the daily published Moroccan data [14], we estimate the values of some parameters of the model. The proportion of asymptomatic forms can vary from $20.6 \%$ 
of infected population to $39.9 \%$ [15]. Then, $\epsilon \in[0.61,0.794]$. The progression rates $\gamma_{b}, \gamma_{g}$ and $\gamma_{c}$ from symptomatic infected individuals to the three forms are assumed to be $80 \%$ of diagnosed cases for benign form, $15 \%$ of diagnosed cases for severe form, and $5 \%$ of diagnosed cases for critical form, respectively [16]. The true mortality of COVID-19 will take some time to be fully understood. The data we have so far indicate that the crude mortality ratio (the number of reported deaths divided by the reported cases) is between 3 and 4\% [16]. As the Moroccan health system is not overloaded at the moment, it is assumed that deaths mainly come from critical cases with a percentage of $40 \%$ for an average period of 13.5 days [16]. Since the mortality rate of symptomatic individuals differs from country to country [6], we assume that $1 \%$ of symptomatic individuals die for an average period of 21 days, whereas the recovery rate for asymptomatic cases is $100 \%$ and is the same for severe and benign forms if a proper medical care is taken with an average period of 21 days. We employ a least-square procedure with Poisson noise as in [5] to estimate the transmission rate. The incubation period is estimated to be 5.5 days [17, 18] while the time needed before hospitalization is estimated to be 7.5 days [19, 20, 21]. The estimation of the above parameters is given in Table 1

Table 1: Parameter values for our model 11 .

\begin{tabular}{|c|c|c|}
\hline$\overline{\text { Parameter }}$ & Value & Source \\
\hline$\beta$ & $0.4517(95 \% C I, 0.4484-0.455)$ & Estimated \\
\hline$u$ & $0-1$ & Varied \\
\hline$\epsilon$ & 0.794 & [15] \\
\hline$\gamma_{b}$ & 0.8 & {$[16$} \\
\hline$\gamma_{g}$ & 0.15 & [16] \\
\hline$\gamma_{c}$ & 0.05 & [16] \\
\hline$\alpha$ & 0.06 & Assumed \\
\hline$\eta_{a}$ & $1 / 21$ & Calculated \\
\hline$\eta_{s}$ & $0.8 / 21$ & Calculated \\
\hline$\mu_{s}$ & $0.01 / 21$ & Calculated \\
\hline$\mu_{b}$ & 0 & Assumed \\
\hline$\mu_{g}$ & 0 & Assumed \\
\hline$\mu_{c}$ & $0.4 / 13.5$ & Calculated \\
\hline$r_{b}$ & $1 / 13.5$ & Calculated \\
\hline$r_{g}$ & $1 / 13.5$ & Calculated \\
\hline$r_{c}$ & $0.6 / 13.5$ & Calculated \\
\hline$\tau_{1}$ & 5.5 & [17, 18 \\
\hline$\tau_{2}$ & 7.5 & [19, 20, 21 \\
\hline
\end{tabular}

Sensitivity analysis is commonly used to determine the robustness of model predictions to some parameter values. It is used to discover parameters that have a high impact on $\mathcal{R}_{0}$ and should be targeted by intervention strategies. The main objective of this section is to examine the sensitivity of the basic reproduction number $\mathcal{R}_{0}$ with respect to model parameters by the so-called sensitivity index. 
Definition $1([\mathbf{2 2}, 23])$ The normalized forward sensitivity index of a variable $v$, that depends differentially on a parameter $\rho$, is defined as

$$
\Upsilon_{\rho}^{v}:=\frac{\partial v}{\partial \rho} \times \frac{\rho}{v} .
$$

According to Definition 1 we derive the normalized forward sensitivity index of $\mathcal{R}_{0}$ with respect to $\beta, \epsilon, \eta_{s}, \mu_{s}, \gamma_{b}, \gamma_{g}, \gamma_{c}$, and $\alpha$, which is summarized in Table 2 . As we observe in Table 2, the most sensitive parameters, which have a higher impact

Table 2: The normalized forward sensitivity index of $\mathcal{R}_{0}$.

\begin{tabular}{cccc}
\hline \hline Parameters & Sensitivity index of $\mathcal{R}_{0}$ & Value \\
\hline$\beta$ & & $\Upsilon_{\beta}^{R_{0}}=+1$ & +1 \\
$\epsilon$ & $\Upsilon_{\epsilon}^{R_{0}}=+1$ & +1 \\
$\eta_{s}$ & $\Upsilon_{\eta_{s}}^{R_{0}}=-\frac{\eta_{s}}{\eta_{s}+\mu_{s}+\left(\gamma_{b}+\gamma_{g}+\gamma_{c}\right) \alpha}$ & -0.3864 \\
$\mu_{s}$ & $\Upsilon_{\mu_{s}}^{R_{0}}=-\frac{\mu_{s}}{\eta_{s}+\mu_{s}+\left(\gamma_{b}+\gamma_{g}+\gamma_{c}\right) \alpha}$ & -0.0048 \\
$\gamma_{b}$ & $\Upsilon_{\gamma_{b}}^{R_{0}}=-\frac{\alpha \gamma_{b}}{\eta_{s}+\mu_{s}+\left(\gamma_{b}+\gamma_{g}+\gamma_{c}\right) \alpha}$ & -0.487 \\
$\gamma_{g}$ & $\Upsilon_{\gamma_{g}}^{R_{0}}=-\frac{\alpha \gamma_{g}}{\eta_{s}+\mu_{s}+\left(\gamma_{b}+\gamma_{g}+\gamma_{c}\right) \alpha}$ & -0.0913 \\
$\gamma_{c}$ & $\Upsilon_{\gamma_{c}}^{R_{0}}=-\frac{\alpha \gamma_{c}}{\eta_{s}+\mu_{s}+\left(\gamma_{b}+\gamma_{g}+\gamma_{c}\right) \alpha}$ & -0.0304 \\
$\alpha$ & $\Upsilon_{\alpha}^{R_{0}}=-\frac{\alpha\left(\gamma_{b}+\gamma_{g}+\gamma_{c}\right)}{\eta_{s}+\mu_{s}+\left(\gamma_{b}+\gamma_{g}+\gamma_{c}\right) \alpha}$ & -0.687 \\
\hline \hline
\end{tabular}

on $\mathcal{R}_{0}$, are $\beta$ and $\epsilon$, since $\Upsilon_{\beta}^{\mathcal{R}_{0}}$ and $\Upsilon_{\epsilon}^{\mathcal{R}_{0}}$ are independent of any parameter of system 11 with $\Upsilon_{\beta}^{\mathcal{R}_{0}}=\Upsilon_{\epsilon}^{\mathcal{R}_{0}}=+1$. In addition, the parameter $\alpha$ has a middle negative impact on $\mathcal{R}_{0}$, while $\mathcal{R}_{0}$ is slightly impacted by the rest of the parameters.

\section{Prevision of COVID-19 in Morocco}

In this section, we present the forecasts of COVID-19 in Morocco relating with different preventive measures and strategies implemented by Moroccan authorities on the confinement period between March 2 and June 20,2020. Then the parameter $u$ can be defined as follows: 


$$
u=\left\{\begin{array}{l}
u_{1}, \text { on } \text { (March 2, March 10]; } \\
u_{2}, \text { on (March 10, March 20]; } \\
u_{3}, \text { on (March 20, April 6]; } \\
u_{4}, \text { after April 6, }
\end{array}\right.
$$

where $u_{i} \in(0,1], i=1,2,3,4$, measures the effectiveness of applying the multiple preventive interventions imposed by Moroccan authorities presented in Table 3

Table 3: Summary of non-pharmaceutical interventions considered.

\begin{tabular}{ll}
\hline \hline Policies & Control values \\
\hline Without any intervention measures & $u=0$, after March 2 \\
\hline First set of measures & $u=0.2$, after March 2 \\
\hline Second set of measures & $u=0.2$, on (March 2, March 10] and \\
& $u=0.3$, after March 10 \\
\hline Third set of measures & $u=0.2$, on (March 2, March 10], \\
& $u=0.3$, on (March 10, March 16] and \\
& $u=0.4$, after March 16 \\
\hline Fourth set of measures & $u=0.2$, on (March 2, March 10], \\
& $u=0.3$, on (March 10, March 16] and \\
$u$ & $=0.4$, on (March 16, April 6] and \\
$u$ & $u=0.8$, after April 6, \\
\hline \hline
\end{tabular}

To make a better illustration of the different strategies, we test the four decisions made at the government level in Figure 2

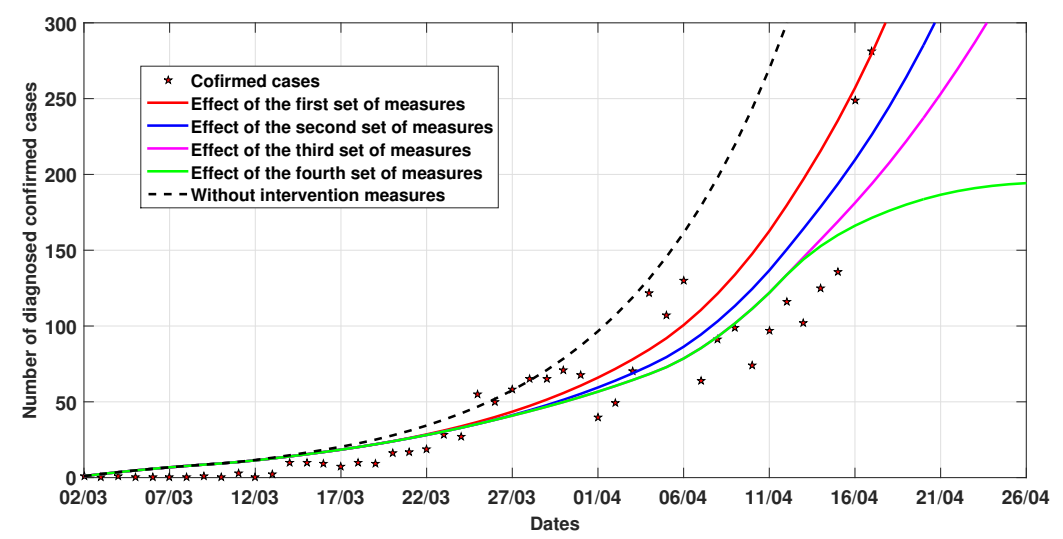

Fig. 2: Comparison of the non-pharmaceutical interventions considered and the daily reported cases of COVID-19 in Morocco from March 2 to April 17, 2020. 
We see in Figure 2 the evolution of the number of diagnosed infected positive individuals with different sets of measures: low, middle, high, and strict interventions. Up to April 15, the curves corresponding to the first three sets of measures increase exponentially, while the curve corresponding to the fourth set of measures has lost its initial exponential character and tends to flatten over time. In addition, the last daily reported cases in Morocco from March 2 to April 17, confirm the biological tendency of our model. Thus, our model is efficient to describe the spread of COVID-19 in Morocco. However, we note that some clinical data is a little far from the values of the model due to certain foci that appeared in some large areas or at the level of certain industrial areas.

Next, we give the graphical results related to delays parameters to prove their biological importance.

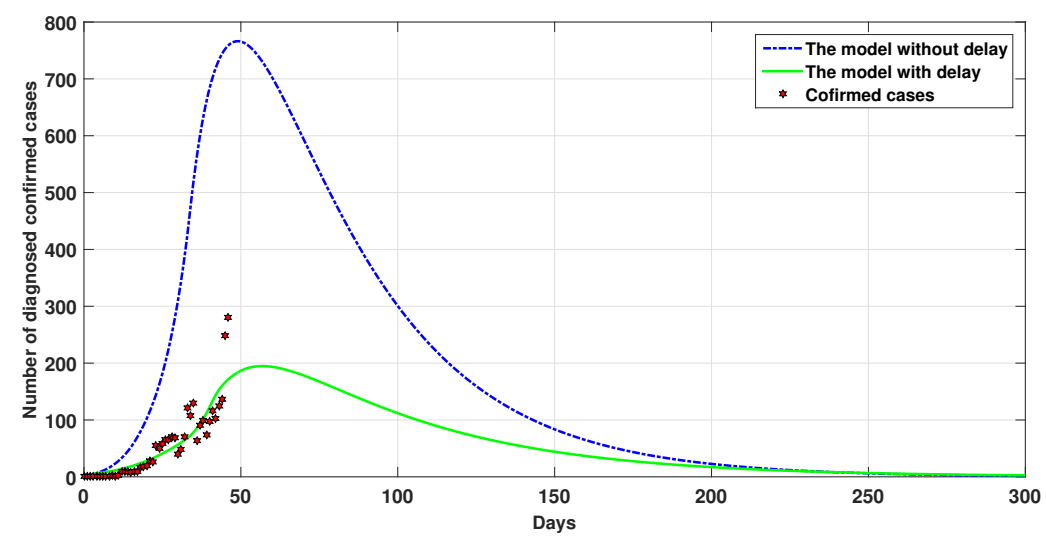

Fig. 3: Effect of delays on the diagnosed confirmed cases.

We observe in Figure 3 a highly impact of delays on the number of diagnosed positive cases, thereby the plot of model (1) without delays $\left(\tau_{1}=\tau_{2}=0\right)$ is very far from the clinical data.

\subsection{Peak prediction}

Now, we indicate the predicted relative impact of the model and especially the diagnosed infective individuals with and without interventions applied progressively in Morocco. 
Before finding the first positive infected case in Morocco, the authorities have begun with a suspension of international air lines to and from China, and installed health control check-points at the borders but without any interventions into the Moroccan population. For this, we simulate model (1) in the case $u=0$, which is illustrated by Figures 4 and 5
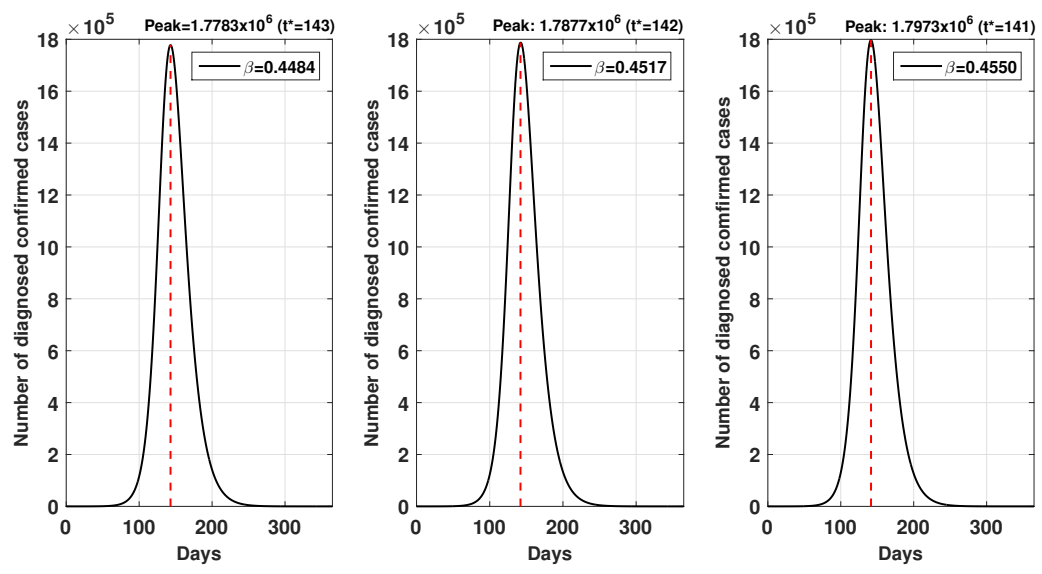

Fig. 4: Time variation of the diagnosed infective individuals without any intervention on the Moroccan population with different values of $\beta(95 \% C I, 0.4484-0.455)$.

We remark from Figure 4 that the estimated epidemic peak is $t^{*}=142$ $(95 \% C I, 141-143)$, that is, starting from March 2, $2020(t=0)$, the estimated epidemic peak is July $21,2020(t=142)$.

In the absence of any government intervention, the disease persists strongly and almost all of the vulnerable population will be reached by the infection (Figure 57.

After the first imported positive infected case, Moroccan authorities began to establish some preventive interventions between the 2 and the 10th of March, namely isolation of positive cases, contact tracing, hygiene measures, prevention measures in workplaces, and ban of mass gathering events. For this reason, we have selected in this period $u=0.2$. From March 10 up to March 20, 2020, additional preventive measures were established: gradual suspension of all international sea, ground, and air lines (including with Spain, Italia, Algeria, France, Germany, Netherlands, Belgium, and Portugal), closure of coffees, restaurants, cinemas, theaters, party rooms, clubs, sport centers, hammams, game rooms and sport fields, closure of mosques, schools and universities, disinfection of public transportation means, reduction of the carrying capacity of taxis, buses and tramways, movement/travel restrictions, and containment 

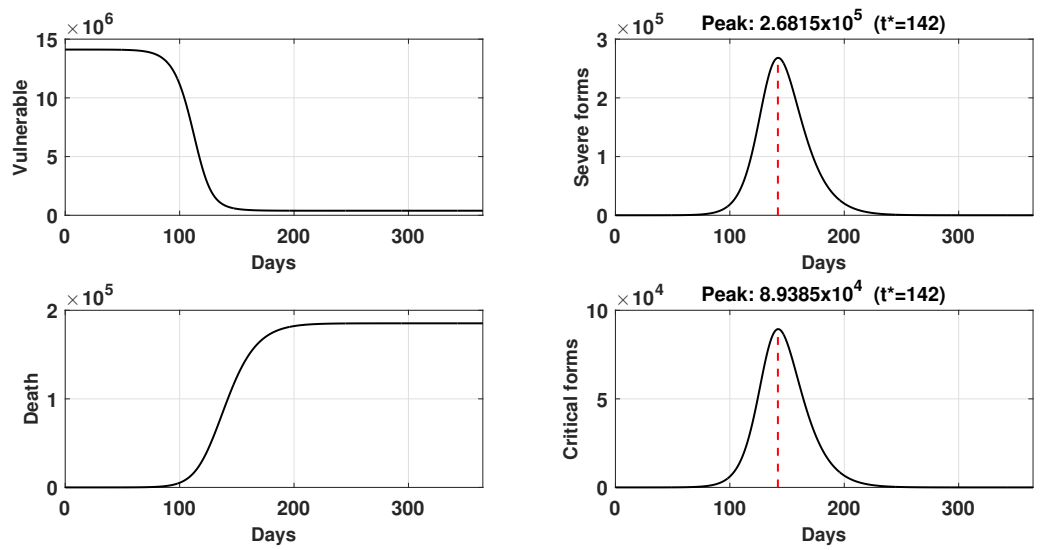

Fig. 5: Time variation of the model with $\beta=0.4517$ and $\mathcal{R}_{0}=3.6385$.

measures of the general population. These measures correspond to the choice of the control $u=0.3$. From March 20 up to April 6, the Moroccan authority declared a state of emergency with a complete lockdown, night-time curfew, movement restrictions $24 / 24$, ban of human movements between cities, suspension of railway lines, streets disinfection, and extensive cleaning and disinfection of port and airport facilities. For this, we assume that $u=0.4$. From April 6, the authority decided compulsory wearing of masks in public spaces, which implies a significant positive influence on the above interventions and an increase of their efficiency level. In this case, we assume that $u=0.8$. Tacking into account all these policies, we present the following Figures 6 and 7

We remark from Figure 6 that the estimated epidemic peak is $t^{*}=57(95 \% C I, 56-$ 57), that is, starting from March $2(t=0)$, the estimated epidemic peak is April 28, $2020(t=57)$.

From Figure 7, we see that all the measures taken into this second strategy have a significant impact on the number of new positive diagnosed cases per day. Compared to Figure 5 , the time required to reach the peak is reduced by 85 days, avoiding globally an interesting number of new infections and new deaths. Furthermore, the computed basic reproduction number $\mathcal{R}_{0}$ is less than 1 , which means the extinction of the disease if the measures cited above are strictly implemented. 

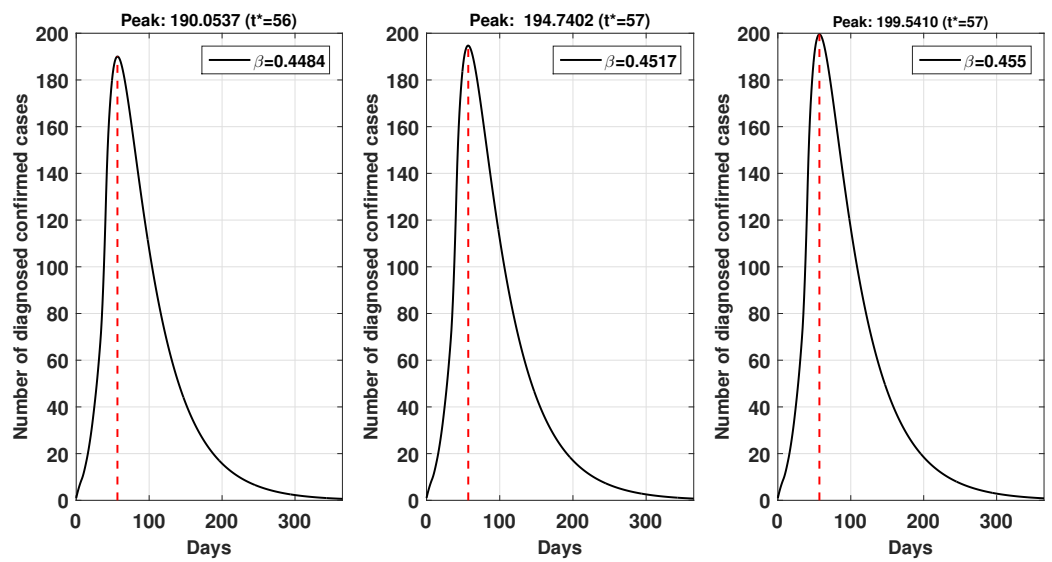

Fig. 6: Time variation of the diagnosed infective individuals with hight level respect of measures for different values of $\beta(95 \% C I, 0.4484-0.455)$.
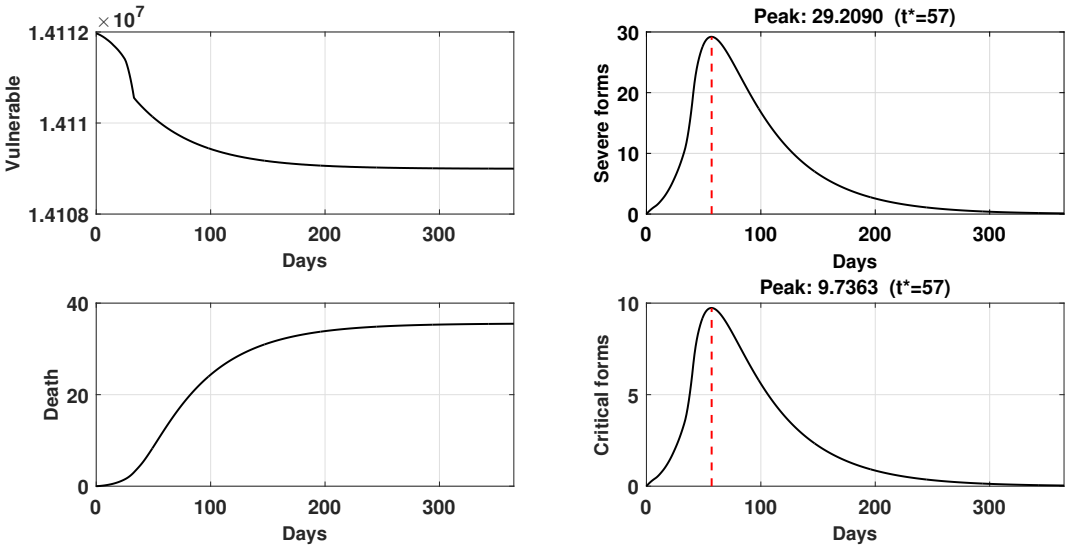

Fig. 7: Time variation of the model with $\beta=0.4517$ and $\mathcal{R}_{0}=2.9108$ (March 2-10), $\mathcal{R}_{0}=2.5469$ (March 10-20), $\mathcal{R}_{0}=2.1831$ (March 20-April 6), $\mathcal{R}_{0}=0.7277$ (from April 6, 2020).

\subsection{Intervention effectiveness}

Here, on one hand, we compare the impact of different degrees of effectiveness on the evolution of the number of positive infected diagnosed individuals, symptomatic individuals, and deaths (see Figures 8 and 9 ). In addition, we present the cumulative cases in Figure 10 and we summarize it in Table 4 . We remark that the effectiveness of the policies plays an important role to reduce, or not, the human damage and 
ensure the eradication of the illness. However, mitigation measures must be strictly respected to maintain a good level of control over the spread of the virus.

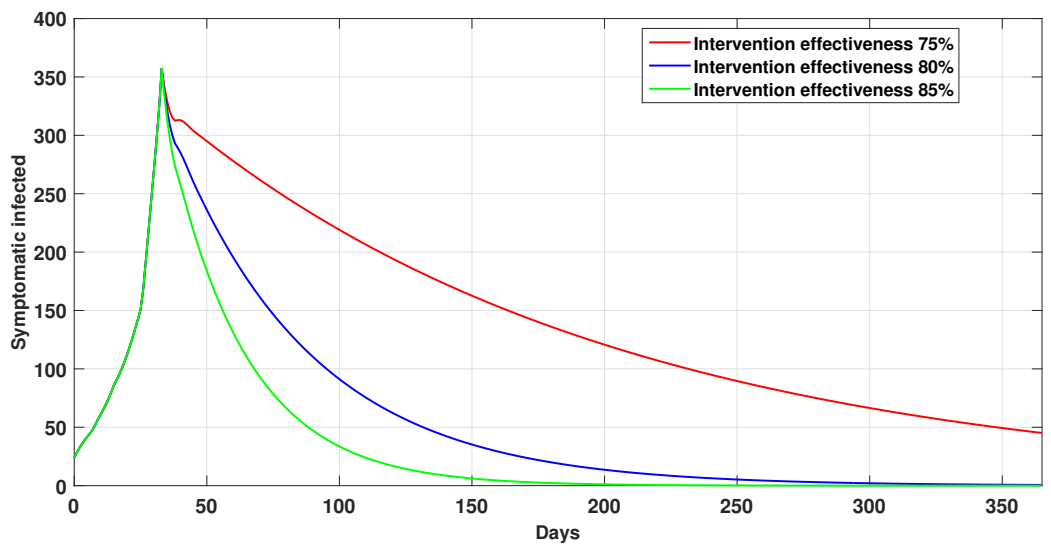

Fig. 8: Evolution of the symptomatic individuals with different effectiveness degrees.
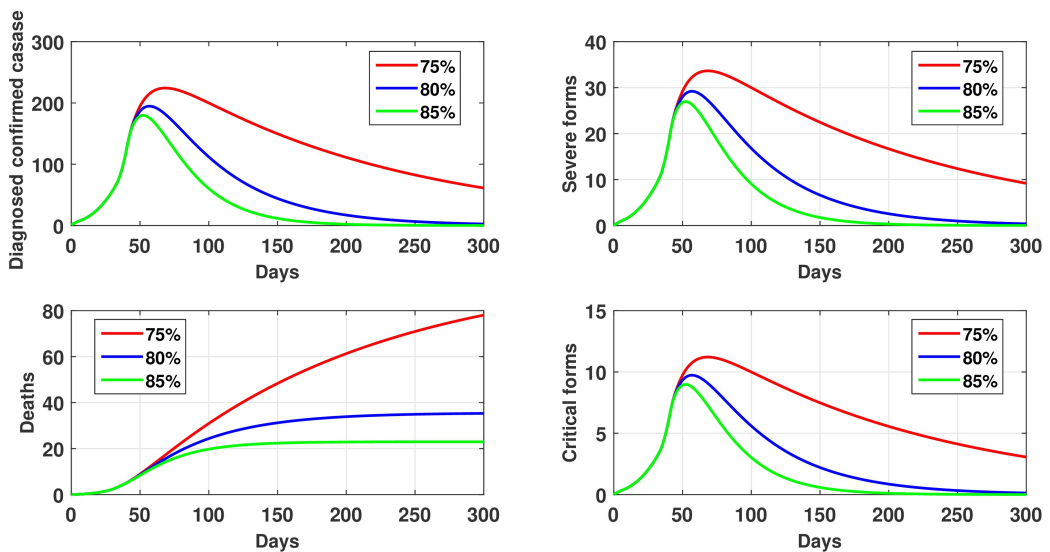

Fig. 9: Evolution of the positive infected diagnosed individuals and deaths with different effectiveness degrees. 

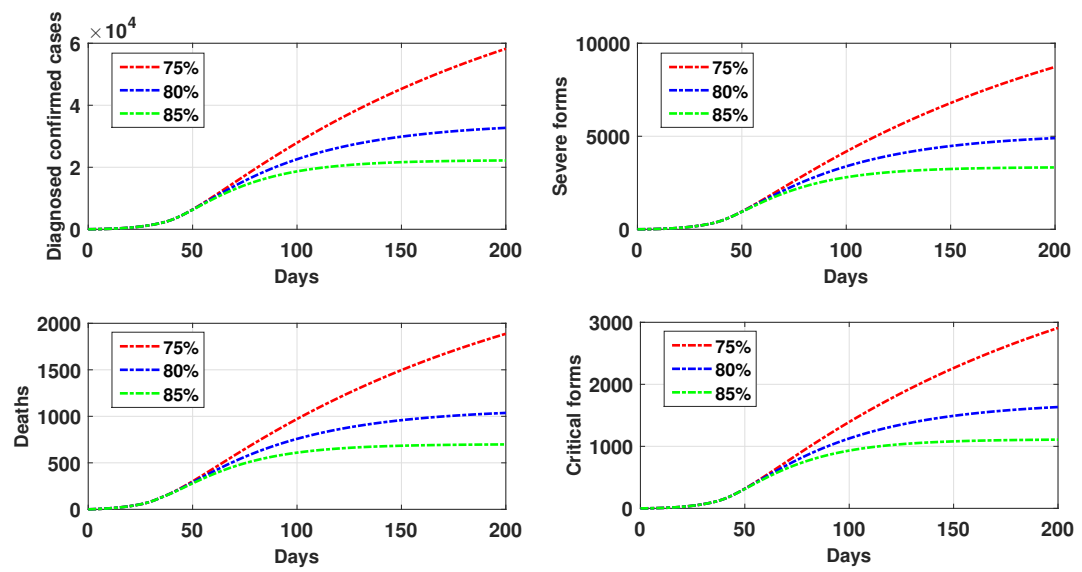

Fig. 10: Cumulative diagnosed cases, severe forms, critical forms, and deaths, with different effectiveness degrees.

Table 4: Cumulative diagnosed cases, severe forms, critical forms, and deaths, after 150 days of the start of the pandemic in Morocco.

\begin{tabular}{lccc}
\hline \hline Effectiveness & $75 \%$ & $80 \%$ & $85 \%$ \\
\hline \hline Diagnosed & 42834 & 29116 & 21432 \\
\hline Severe forms & 6419 & 4361 & 3209 \\
\hline Critical forms & 2139 & 1453 & 1069 \\
\hline Deaths & 1500 & 993 & 661 \\
\hline \hline
\end{tabular}

On the other hand, we are carrying out a statistical study on a national scale and we note that the trend at the beginning was exponential and will undergo a break due to the multiple interventions of the government, which is globally a good sign (see Figure 11, whereas it is needful to pay attention at the evolution of the curves in the different regions in Morocco. Since the clinical data of COVID-19 was not available on a daily basis at the start of the spread of the epidemic in Morocco, we proceeded with a choice of unit of three days. We also remark that almost all the regions have a homogeneous tendency with the national one, except Tangier-Tetouan-Al Hoceima (TTA), Oriental, Marrakech-Safi (MS), and Casablanca-Settat (CS), which show a mitigation of the epidemic that does not seem very stable (see Figures 12 and 13). 


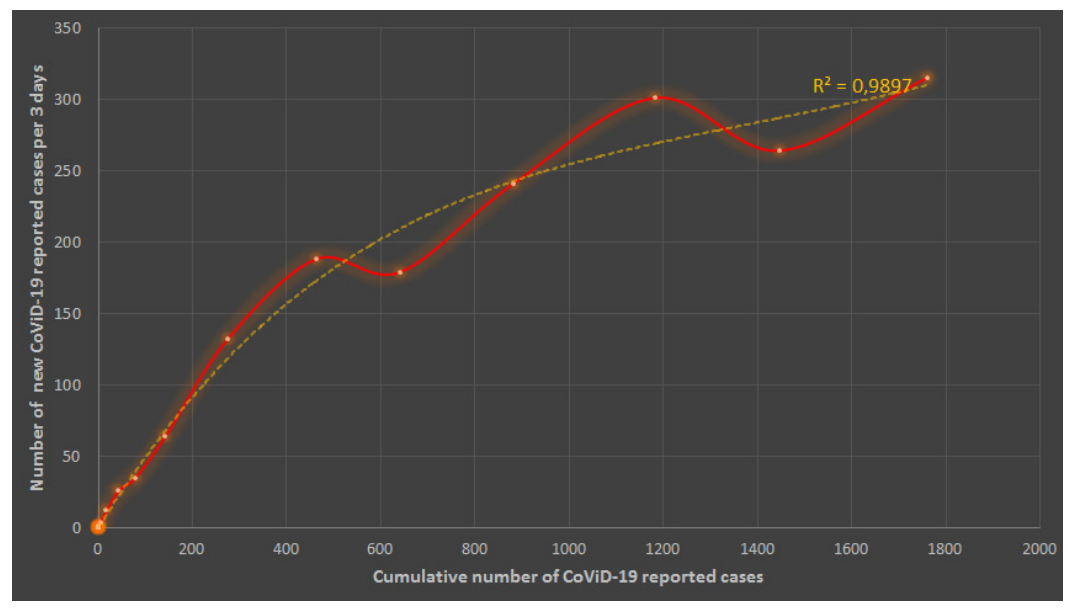

Fig. 11: Trends in the number of new COVID-19 reported cases per three days in Morocco, compared to the cumulative number of COVID-19 reported cases with correlation coefficient $R^{2}=0.9897$.

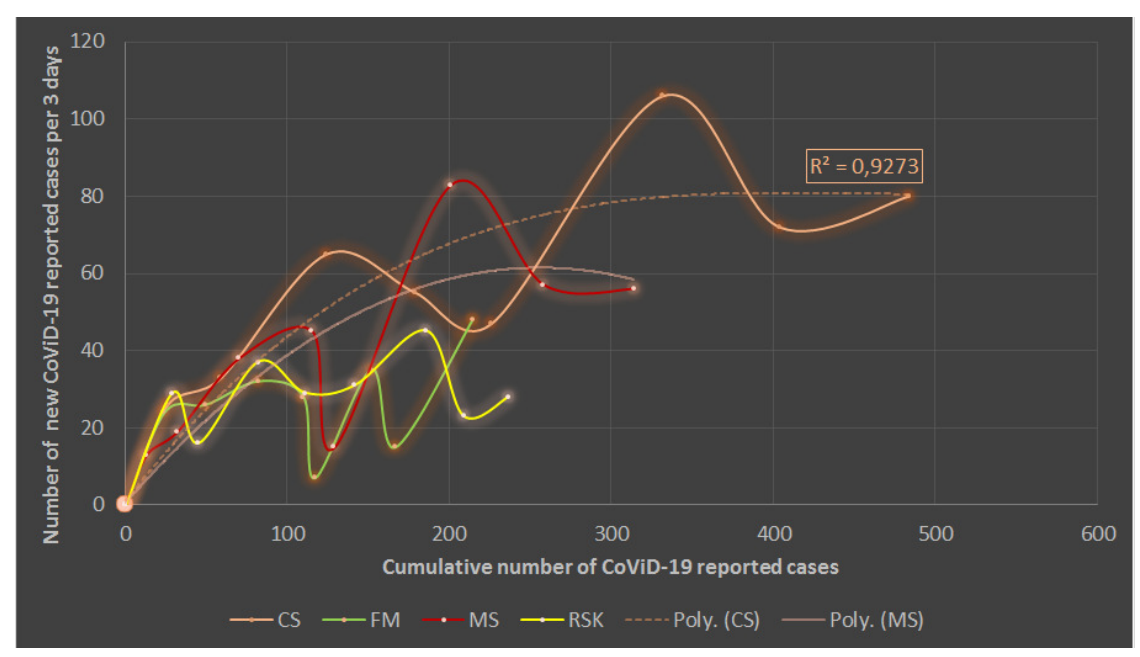

Fig. 12: Trends in the number of new COVID-19 reported cases per three days in Morocco, by regions, compared to the cumulative number of COVID-19 reported cases (CS: Casablanca-Settat; FM: Fes-Meknes; MS: Marrakech-Safi; RSK: RabatSale-Kenitra). 


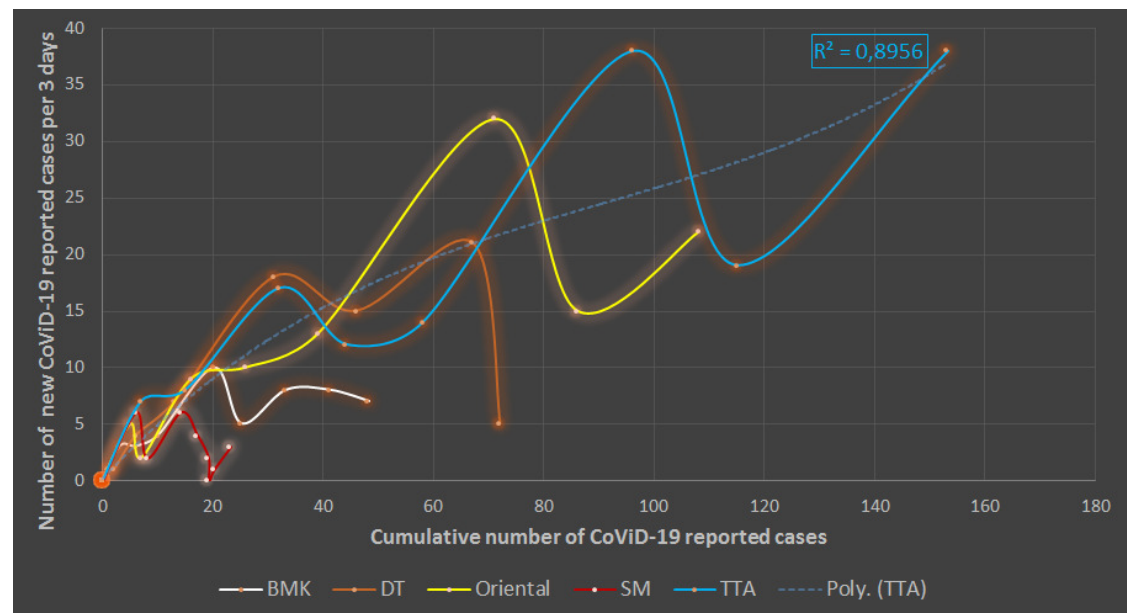

Fig. 13: Trends in the number of new COVID-19 reported cases per three days in Morocco, by regions, compared to the cumulative number of COVID-19 reported cases (BMK: Beni Mellal-Khenifra; DT: Daraa-Tafilalet; SM: Souss-Massa; TTA: Tetouan-Tangier-Assillah).

\section{Discussion}

Our work followed several steps. First of all, we have formulated an adequate mathematical model to describe the evolution of the COVID-19 disease epidemic in Morocco. This model allows us to have an idea on the number of resuscitation beds to prepare for severe forms and intensive care units for critical forms. Second, we have estimated the corresponding parameters based on the daily Moroccan data. Furthermore, we have applied the least-square-method to determine the confidence interval of the transmission rate $\beta$, which is given by $(95 \% C I, 0.4484-0.455)$. Third, we have computed the basic reproduction number $\mathcal{R}_{0}$ with the next-generation-matrix method, for which we have studied the sensitivity analysis in order to examine the robustness of the model. We have observed that the transmission rate $\beta$ and the proportion of individuals with symptoms $\epsilon$ are the most sensitive parameters and have a high impact on $\mathcal{R}_{0}$. By performing some numerical simulations, we have represented the effect of measures taken by the government, step by step, with the control $u$. In the first period, the appropriate basic reproduction number for $u=0.2$ is $\mathcal{R}_{0}=2.9108$. In the second period, $\mathcal{R}_{0}=2.5469$ for $u=0.3$. Thirdly, $\mathcal{R}_{0}=2.1831$ for $u=0.4$. In the last period, $\mathcal{R}_{0}=0.7277$ for $u=0.8$. Based on all strategies taken by the Moroccan authorities, we affirm that the best one is to increase considerably the level of the lockdown accompanied by the general use of the face masks. In this case, the estimated endemic peak will take place around April 28. Finally, through an analysis of regional data, we have shown that the evolution of the pandemic is consistent with the general epidemiological tendency at the national level. 
We finish by mentioning that the used historic data and the different preventive measures and strategies implemented by Moroccan authorities and considered in our study, are related to the confinement period in Morocco, between March 2 and June 20, 2020, whereas the use of historic data and other measures and strategies, linked to the deconfinement phase, are left to another research work.

Acknowledgements HZ and DFMT were supported by FCT within project UIDB/04106/2020 (CIDMA). YA brought his expertise in the realization of this work but this does not, in any way, reflects an opinion of the World Health Organization (WHO). The authors would like to express their gratitude to the anonymous reviewers for their constructive comments and suggestions, which helped them to enrich the work.

\section{References}

1. A. E. Gorbalenya, S. C. Baker, R. S. Baric, R. J. de Groot, C. Drosten, A. A. Gulyaeva, B. L. Haagmans, C. Lauber, A. M. Leontovich, B. W. Neuman, D. Penzar, S. Perlman, L. L. M. Poon, D. V. Samborskiy, I. A. Sidorov, I. Sola and J. Ziebuhr, The species Severe acute respiratory syndromerelated coronavirus: classifying 2019-nCoV and naming it SARS-CoV-2, Nature Microbiology 5 (2020), 536-544.

2. Ministry of Health of Morocco, The official portal of Corona virus in Morocco. Available online, http://www.covidmaroc.ma/pages/Accueil.aspx

3. B. Tang, X. Wang, Q. Li, N. L. Bragazzi, S. Tang, Y. Xiao and J. Wu, Estimation of the transmission risk of the 2019-nCoV and its implication for public health interventions, Journal of Clinical Medicine 9 (2020), no. 2, Art. 462, 13 pp.

4. J. T. Wu, K. Leung and G. M. Leung, Nowcasting and forecasting the potential domestic and international spread of the 2019-nCoV outbreak originating in Wuhan, China: a modelling study, The Lancet 395 (2020), no. 10225, 689-697.

5. T. Kuniya, Prediction of the Epidemic Peak of Coronavirus Disease in Japan, 2020, Journal of Clinical Medicine 9 (3) (2020), Art. 789, 7 pp.

6. D. Fanelli and F. Piazza, Analysis and forecast of COVID-19 spreading in China, Italy and France, Chaos, Solitons \& Fractals 134 (2020), Art. 109761, 5 pp.

7. F. Ndaïrou, I. Area, J. J. Nieto and D. F. M. Torres, Mathematical modeling of COVID-19 transmission dynamics with a case study of Wuhan, Chaos Solitons Fractals 135 (2020), Art. 109846, 6 pp. arXiv:2004.10885

8. K. M. Altaf and A. Atangana, Modeling the dynamics of novel coronavirus (2019-nCov) with fractional derivative, Alexandria Engineering Journal 59 (2020), no. 4, 2379-2389.

9. M. L. Bell, A. Zanobetti and F. Dominici, Evidence on vulnerability and susceptibility to health risks associated with short-term exposure to particulate matter: A systematic review and meta-analysis, American Journal of Epidemiology 178 (2013), no. 6, 865-876.

10. M. H. Kottow, The vulnerable and the susceptible, Bioethics 17 (2003), no. 5-6, 460-471.

11. J. K. Hale and S. M. Verduyn Lunel, Introduction to functional-differential equations, Applied Mathematical Sciences, 99, Springer-Verlag, New York, 1993.

12. O. Diekmann, J. A. P. Heesterbeek and J. A. J. Metz, On the definition and the computation of the basic reproduction ratio $R_{0}$ in models for infectious diseases in heterogeneous populations, J. Math. Biol. 28 (1990), 365-382.

13. P. V. Driessche and J. Watmough, Reproduction numbers and sub-threshold endemic equilibria for compartmental models of disease transmission, Math. Biosci. 180 (2002), 29-48.

14. Ministry of Health, Morocco, Department of Epidemiology and Disease Control, http:// wWW.sante.gov.ma/Pages/Accueil.aspx 
15. K. Mizumoto, K. Kagaya, A. Zarebski and G. Chowell, Estimating the asymptomatic proportion of coronavirus disease 2019 (COVID-19) cases on board the Diamond Princess cruise ship, Yokohama, Japan, 2020, Eurosurveillance 25 (2020), no. 10, Art. 2000180, 5 pp.

16. WHO, Coronavirus disease 2019 (COVID-19), Situation Report 46, 6 March 2020.

17. WHO, Coronavirus disease 2019 (COVID-19), Situation Report 73, 2nd April 2020.

18. S. G. Baum, COVID-19 Incubation Period: An Update. March 13, 2020, https://www. jwatch.org/na51083/2020/03/13/covid-19-incubation-period-update

19. C. Huang et al., Clinical features of patients infected with 2019 novel coronavirus in Wuhan, China, Lancet 395 (2020), no. 10223, 497-506.

20. D. Wang et al., Clinical characteristics of 138 hospitalized patients with 2019 novel Coronavirus-Infected pneumonia in Wuhan, China, JAMA 323 (2020), no. 11, 1061-1069.

21. Haut Conseil de la Santé publique, Morocco, avis relatif aux recommandations thérapeutiques dans la prise en charge du COVID-19 (complémentaire à l'avis du 5 mars 2020), 23 mars 2020.

22. N. Chitnis, J. M. Hyman and J. M. Cushing, Determining important parameters in the spread of malaria through the sensitivity analysis of a mathematical model, Bull. Math. Biol. 70 (2008), no. 5, 1272-1296.

23. H. S. Rodrigues, M. T. T. Monteiro and D. F. M. Torres, Sensitivity analysis in a dengue epidemiological model, Conf. Papers in Math. 2013 (2013), Art. 721406, 7 pp. arXiv: 1307.0202 